\title{
JEU VIDÉO D'APPRENTISSAGE DES LANGUES EN MILIEU SCOLAIRE : CE QU'EN DISENT LES ÉLÈVES
}

\author{
Laurence SCHMOLL ${ }^{1}$
}

Les relations entre le jeu et le milieu scolaire sont tissées de tensions et d'incompréhensions, alors même qu'un certain nombre de rapprochements sont possibles, notamment dans le domaine de l'apprentissage des langues. Cet article se propose d'étudier le discours d'élèves de seconde quant aux notions de jeu et de jeu vidéo d'apprentissage suite à leur immersion dans un dispositif ludo-éduquant pour l'apprentissage de l'allemand, dans le but de déterminer quels facteurs peuvent représenter un atout ou un frein à la médiation ludo-éducative en milieu scolaire. Nous analyserons ainsi en quoi le dispositif proposé ainsi que le contexte scolaire influencent les représentations des élèves et si, de ce fait, son emploi est pertinent au regard des premiers concernés.

En faisant référence à Anderson $(1988$, p. 11) et en évoquant sa définition du média comme « une activité humaine distincte qui organise la réalité en textes lisibles en vue de l'action », l'appel à contribution de ce numéro place le jeu dans le cadre d'analyse des théories de l'action. Ce positionnement est particulièrement pertinent dans le domaine de la didactique des langues et des cultures,

1 Laurence Schmoll est enseignante à la Faculté des Lettres et au Département de Linguistique Appliquée et de Didactique des Langues (Dladl) de l'Université de Strasbourg et docteure en Sciences du langage au sein de l'Équipe d'Accueil Linguistique, Langues, Parole (EA 1339 LiLPa).

Recherches en communication, $\mathrm{n}^{\circ} 49$ - Article publié le 21/03/2019 
car l'orientation méthodologique actuelle, à savoir la perspective actionnelle, considère les apprenants « comme des acteurs sociaux ayant à accomplir des tâches (qui ne sont pas seulement langagières) dans des circonstances et un environnement donnés, à l'intérieur d'un domaine d'action particulier » (Conseil de l'Europe, 2001, p. 15). Nous percevons donc un lien relativement fort entre jeu et apprentissage des langues, le jeu étant considéré dans un contexte d'apprentissage des langues comme un artéfact qui joue « un rôle de médiateur entre le sujet et l'objet de son activité » (Meunier \& Peraya, 2010, p. 368). Cette médiation ne va cependant pas de soi, étant donné qu'elle dépend du positionnement de l'utilisateur, non seulement face à l'objet présenté comme un jeu (Henriot, 1969), mais aussi face à l'environnement dans lequel il est proposé, ici la classe de langue et, par extension, le contexte scolaire.

Le processus de formalisation (Brougère, 2002) par lequel passe le jeu dès lors qu'il est pensé dans une visée éducative n'est pas sans soulever certains problèmes. Plusieurs recherches se sont penchées sur le jeu vidéo en tant qu'objet d'apprentissage des langues (par exemple, Sykes \& Reinhardt, 2013 ; Schmoll, 2016a), mettant souvent en avant les difficultés à concilier les deux dimensions opposées qui le caractérisent : les ressorts ludiques d'une part et l'intention sérieuse d'autre part (Alvarez, 2007). D'autres se sont intéressés à son intégration dans la classe en interrogeant les pratiques des enseignants et leurs ressentis quant à ses apports et ses limites pédagogiques (Wastiau, 2009 ; Wix, 2012). Concernant les principaux utilisateurs concernés, les apprenants, les études qui s'y intéressent orientent surtout leur recherche sur les pratiques de ces derniers, l'accueil qu'ils peuvent faire au dispositif ludo-éduquant proposé ou encore leurs ressentis quant à un possible apprentissage (Wix, 2012 par exemple). Elles ne font cependant pas forcément le lien avec les représentations que les apprenants ont du jeu au départ et, de ce fait, les raisons pour lesquelles ils considèrent que l'objet ou l'activité qui leur est proposé est un jeu.

Nous souhaitons ainsi nous concentrer plus précisément sur le discours des élèves et leurs représentations à ce sujet. Le cor- 
pus est composé d'entretiens menés auprès d'élèves de seconde ayant testé un dispositif ludo-éduquant ${ }^{2}$ pour l'apprentissage de l'allemand proposé dans le cadre d'une expérimentation en milieu scolaire. Quels sont les attributs que les élèves confèrent au jeu (vidéo) ? Considèrent-ils le dispositif proposé comme un jeu et pour quelles raisons ? Dans quelle mesure le contexte d'enseignement-apprentissage influence-t-il leur point de vue sur ce dernier? Subséquemment, le développement d'une attitude ludique en contexte scolaire est-il envisageable ? Et à quelles conditions?

\section{Jeu et apprentissage des langues}

Henriot (1969) distingue trois niveaux dans la définition du jeu : l'objet ou l'activité nommés comme tels par un groupe donné, l'activité en elle-même et enfin l'attitude mentale dans laquelle se place l'individu lorsqu'il se trouve face à l'objet et que le philosophe nomme « attitude ludique » (Henriot, 1969, p. 73). Il propose ainsi une définition du jeu centrée sur le sujet jouant, car c'est lui qui choisit ce qui est un jeu ou ce qui ne l'est pas, en fonction de l'objet présenté, de son état d'esprit, de l'environnement immédiat dans lequel il est intégré, ainsi que du contexte socio-culturel dont il est imprégné. Ces variables sont à prendre en considération dans la conception et l'intégration d'un jeu à visée pédagogique en milieu scolaire (Schmoll, 2016a). En effet, ces différents facteurs peuvent influer sur le succès d'un tel dispositif auprès de son public cible.

La définition du jeu proposée par Henriot représente un autre intérêt dans la mesure où la centration sur le sujet jouant rejoint celle de centration sur l'apprenant-utilisateur de la langue développée dans la perspective actionnelle (Conseil de l'Europe, 2001). En effet, l'apprenant est désormais considéré comme un individu agissant dans la société et dans le monde et non plus seulement dans son apprentissage (Puren, 2009). Il s'agit ainsi de se représenter l'individu comme ayant des objectifs, des besoins,

2 Nous empruntons l'expression à Alvarez (2007, p. 126) qui parle de dispositif ludo-éduquant pour faire référence à l'espace de rencontre du jeu et de l'apprentissage à l'intérieur duquel le tuteur et l'apprenant interagissent. 
des centres d'intérêt et une vie qui ne concernent pas uniquement l'espace-temps de la classe. À ce titre, il est susceptible d'être confronté à des situations de réception ou d'interaction dans la langue-cible en dehors des cours, que ce soit dans la rue s'il se trouve en milieu homoglotte ou par l'intermédiaire de médias tels que le journal, la télévision, Internet ou encore le jeu, et plus particulièrement le jeu vidéo.

En suivant la perspective d'Henriot, nous avons montré dans une précédente étude (Schmoll, 2017) l'intérêt qu'il y a à employer le jeu en classe de langue dès lors que le sujet et l'action sont placés au cœur du dispositif ludo-éduquant. En effet, parce qu'il s'agit d'une activité fictive ou « de second degré » (Brougère, 2005), l'apprenant intègre un espace-temps qui n'est pas celui de la réalité dans lequel il peut prendre des risques, ici langagiers, tout en préservant sa face au cours d'une interaction qui ne présente de danger que pour la réussite ou l'échec de la partie. Il est donc susceptible d'oser davantage s'exprimer. Par ailleurs, l'immersion ludique place le joueur dans une position active qui l'amène à découvrir et apprendre à travers l'observation, l'imitation, l'essai, l'erreur, etc., tout comme il le ferait dans la vie réelle en situation de bain linguistique. Cet apprentissage de type constructiviste n'est cependant possible que lorsque le dispositif respecte le deuxième critère du jeu selon Brougère $(2005)^{3}$, à savoir offrir un espace dans lequel le joueur peut prendre des décisions. Cet espace de liberté représente un des points durs de la conception de jeu vidéo pour l'apprentissage, car il faut réussir à concilier, d'une part, l'expérience ludique du joueur-apprenant qui, s'il a trop de liberté, risque de ne pas savoir quoi faire ou de sortir du cadre préétabli et, d'autre part, un emploi pertinent de la langue-cible en fonction des objectifs et contenus pédagogiques définis.

3 Brougère (2005) évoque cinq critères du jeu. Les deux principaux correspondent à ceux que nous avons déjà évoqués : une activité de second degré et un espace décisionnel. Les trois autres concernent la présence de règles, une incertitude quant à la fin et la frivolité. 


\section{Freins à la médiation ludo-éducative}

À travers le jeu d'apprentissage, les concepteurs, mais aussi les enseignants qui l'utilisent, doivent ainsi réussir non seulement à véhiculer l'idée de jeu (Genvo, 2012), mais aussi à transmettre un message et des contenus pédagogiques. Cette médiation ludoéducative relève cependant d'une double opposition.

La première concerne le dispositif lui-même. Le jeu d'apprentissage est en effet le lieu d'un conflit lié aux représentations que les différentes parties prenantes ont des deux dimensions qu'englobe le concept. La seconde, en lien avec la première, concerne la présence d'un objet issu des loisirs dans un environnement où ces derniers n'ont traditionnellement pas leur place.

Les concepts de jeu et de travail sont historiquement opposés dans les définitions essentialistes du jeu (Caillois, 1967, notamment) et cette idée persiste encore dans certaines cultures ludiques et/ou d'apprentissage (Brougère, 2005). Même s'il existe des exemples d'utilisation de jeux dans le milieu éducatif, ces pratiques représentent souvent un prétexte pour conférer une coloration ludique à un exercice scolaire plutôt qu'une véritable activité ludique (Schmoll, 2016b). Pourtant, de nombreuses recherches, de Piaget à Brougère, en passant par les travaux de l'Éducation nouvelle et les études plus récentes sur l'apprentissage informel (par exemple, Peterson, 2013), mettent en avant le lien entre jeu et apprentissage. Par ailleurs, le jeu, s'il est adapté et intégré de manière réfléchie au dispositif d'enseignement-apprentissage, est susceptible de provoquer davantage d'implication et de motivation chez les apprenants (Wastiau, 2009).

Toutefois, les enseignants et les institutions scolaires continuent à se montrer frileux quant à l'utilisation du jeu dans une perspective pédagogique. Un juste équilibre semble ainsi complexe à trouver pour satisfaire le monde éducatif, et peut-être encore plus les joueurs-apprenants. 


\section{Contexte de la recherche-action}

C'est dans l'intention d'étudier les conditions favorables à l'émergence d'une attitude ludique et d'une implication active (notamment en termes de prise de parole) chez des élèves de seconde apprenant l'allemand langue étrangère au niveau A2 que nous avons, entre 2012 et 2014, participé au développement et à l'évaluation de deux scénarii de jeu vidéo d'apprentissage (Pädagogische Hochschule Karlsruhe, 2011).

L'histoire développée dans le dispositif ludo-éduquant ne revêt que peu d'importance pour cette étude. Il est cependant important de signaler que le matériel employé pour le dispositif technique est conséquent : les élèves sont munis de lunettes 3D et d'un téléphone intelligent auxquels sont fixés des capteurs de mouvement. Ils portent également un casque avec micro afin d'interagir à l'oral avec les Personnages Non Joueurs (PNJ), grâce à la reconnaissance de la parole. Ils font face, en position debout, à un écran de télévision stéréoscopique 3D.

Le premier modèle de scénario (M1) a été conçu selon un séquençage linéaire (Koster, 2005) au cours duquel les joueursapprenants n'ont pas le choix de leurs actions. Ils doivent accomplir leurs tâches dans un ordre préétabli en suivant les consignes et en répondant aux questions d'un PNJ adjuvant qui intervient hors-champ. Les tâches sont donc prescrites.

Suite à l'expérimentation de ce premier scénario auprès de 22 élèves de seconde (T1) et à l'évaluation majoritairement négative qu'ils en ont faite, un deuxième modèle (M2), plus ouvert, a été imaginé. Ce dernier est structuré de façon imbriquée (Koster, 2005), ce qui signifie que les joueurs-apprenants peuvent effectuer un certain nombre de tâches dans l'ordre qu'ils souhaitent. Ces dernières sont plus variées que celles de M1 qui avaient été jugées peu stimulantes tant du point de vue de l'action de jeu que du point de vue cognitif (sélection d'objets et glisser-déposer). Dans M2, les tâches ont donc été réfléchies pour apporter davantage de défis et d'enjeux cognitifs : observation, recherche, sélection, glisser-déposer, résolution d'énigmes, introduction d'un ennemi, évitement. Le PNJ est présent avec eux dans l'environnement et 
commente l'action. Il n'est cependant plus dans un discours prescriptif ou interrogatif. C'est aux joueurs-apprenants de lui poser des questions pour savoir ce qu'ils doivent faire. Les interactions sont donc à l'initiative de l'utilisateur. Le scénario M2 a également fait l'objet d'une expérimentation auprès, cette fois, de 24 élèves de seconde (T2).

\section{Méthodologie}

L'expérimentation a eu lieu, pour T1 et pour T2, au lycée durant les heures de cours, et s'est déroulée en deux temps :

- Une immersion individuelle de 20 minutes filmée. Chaque vidéo a fait l'objet d'une observation post-expérimentation que nous n'exploiterons pas ici.

- Un entretien semi-directif d'une quinzaine de minutes, individuel lui aussi, suivant immédiatement l'immersion. Les entretiens ont été ensuite transcrits et soumis à une analyse de contenu, afin de mettre en exergue les représentations des sujets concernant le dispositif et leur expérience au sein de celui-ci.

Dans le but de déterminer quels facteurs peuvent représenter un atout ou un frein à la médiation ludo-éducative en milieu scolaire, nous nous proposons d'effectuer une analyse du corpus d'entretien en nous axant sur:

1. les représentations des élèves quant au jeu vidéo ;

2. une comparaison des attributs conférés au jeu vidéo (1) avec les raisons qui les mènent à qualifier le dispositif de jeu ou d'une autre manière ;

3. leur avis sur l'utilité d'un tel dispositif pour l'apprentissage de l'allemand.

L'entretien semi-guidé est composé d'une première question ouverte : "Comment c'était ? ", l'objectif étant de laisser parler librement les sujets sans les orienter dans leurs propos. La suite de l'entretien est composée de plusieurs questions destinées notamment à collecter les représentations des sujets quant au dispositif et 
à la notion de jeu vidéo d'apprentissage. Nous nous intéresserons ici principalement à deux questions que nous nommerons successivement (a) et (b).

(a) «Selon toi, comment appellerais-tu ce que tu viens de faire?»

La question est ouverte afin de laisser émerger le plus de qualificatifs possibles en fonction des représentations des sujets. Une reformulation est cependant proposée si la réponse est hors sujet : «Pour toi, est-ce que c'est un jeu, un cours, un exercice, autre chose?

(b) «Que penses-tu de cette idée pour l'apprentissage de l'allemand?»

La question est plus fermée et a pour but d'obtenir une appréciation positive ou négative.

Pour répondre à notre premier point (1), nous nous intéresserons à l'emploi du mot « jeu » par les apprenants dans l'ensemble des entretiens, en dehors des réponses à la question (a). La question (a) sert, quant à elle, à éclairer le point (2). Les réponses à la question (b) nous permettront d'étudier le troisième point (3).

\section{Résultats}

\subsection{Les attributs du jeu (vidéo) dans le discours des élèves}

Nous avons relevé 45 occurrences du terme « jeu » dans l'ensemble du corpus (en dehors des réponses à la question (a) renvoyant à une préconception de la part des sujets quant aux caractéristiques du jeu vidéo). Le vocable est moins présent dans la bouche des élèves ayant expérimenté M1 (scénario linéaire) que dans celle des apprenants ayant testé M2 (scénario imbriqué).

Nous constatons dans le discours des élèves une prépondérance à associer le jeu à des considérations techniques.

Ben déjà en 3D c'était super intéressant euh on est dans le jeu. Ensuite y a, la (inc.) elle est réaliste, j'aime bien les décors mmm, à part quelques bugs mineurs bon ça c'est normal dans les jeux, il était vraiment bien fait quoi. (Wilfred, T2:1) 
Tableau 1. Classification des attributs associés au jeu

\begin{tabular}{|c|c|c|c|}
\hline Catégories & Attributs & $\begin{array}{l}\text { Élèves } \\
\text { de T1 }\end{array}$ & $\begin{array}{l}\text { Élèves } \\
\text { de T2 }\end{array}$ \\
\hline \multirow{3}{*}{$\begin{array}{l}\text { Technique / } \\
\text { technologique } \\
\text { (16 occurrences) }\end{array}$} & $\begin{array}{l}\text { Interactif, beau graphiquement, pré- } \\
\text { sence d'un environnement sonore }\end{array}$ & 3 & 10 \\
\hline & $\begin{array}{l}\text { Présence de boques (considérés comme } \\
\text { normaux) }\end{array}$ & - & 2 \\
\hline & Moderne et modernisant & - & 1 \\
\hline \multirow[t]{2}{*}{$\begin{array}{l}\text { Divertissement } \\
\text { (10 occurrences) }\end{array}$} & $\begin{array}{l}\text { Intéressant, ludique, amusant, prendre } \\
\text { du plaisir }\end{array}$ & 1 & 6 \\
\hline & Temps qui passe plus vite & - & 3 \\
\hline \multirow{3}{*}{$\begin{array}{l}\text { Genre et game ou } \\
\text { level design } \\
\text { ( } 5 \text { occurrences) }\end{array}$} & Aventure, missions, quêtes & 2 & - \\
\hline & Violence, guerre & 2 & - \\
\hline & Inventaire & - & 1 \\
\hline \multirow{2}{*}{$\begin{array}{l}\text { Accessibilité } \\
\text { (4 occurrences) }\end{array}$} & Compréhensible (langue) & 1 & 1 \\
\hline & Compréhensible (scénario) & 1 & 1 \\
\hline \multirow[t]{2}{*}{$\begin{array}{l}\text { Public } \\
\text { (4 occurrences) }\end{array}$} & $\begin{array}{l}\text { Jeunes (les asdolescents jouent aux jeux } \\
\text { vidéo) }\end{array}$ & - & 3 \\
\hline & $\begin{array}{l}\text { Masculin (les hommes sont plus repré- } \\
\text { sentés dans les jeux vidéo) }\end{array}$ & 1 & - \\
\hline $\begin{array}{l}\text { Fictionnel } \\
\text { (2 occurrences) }\end{array}$ & Imaginaire, virtuel, pas la réalité & 1 & 1 \\
\hline \multirow{2}{*}{$\begin{array}{l}\text { Liberté } \\
\text { ( } 2 \text { occurrences) }\end{array}$} & Liberté de choix, autonome & & 1 \\
\hline & Liberté de déplacement, dynamisme & 1 & - \\
\hline $\begin{array}{l}\text { Environnement } \\
(2 \text { occurrences })\end{array}$ & $\begin{array}{l}\text { Hors lycée, pas debout, pas autant } \\
\text { d'équipement) }\end{array}$ & - & 2 \\
\hline
\end{tabular}

Le dispositif technique semble ainsi avoir particulièrement impressionné les sujets qui reconnaissent le matériel (écran, manette, lunettes 3D) comme appartenant à l'univers des jeux vidéo.

Les élèves de T2 parlent également souvent du jeu en y associant la notion de ludique, de divertissement et d'engagement. Nous retrouvons un des critères de Brougère (2005) pour caractériser le jeu : la frivolité, l'idée de pouvoir agir de façon légère, sans conséquence et d'y prendre plaisir. Si ce dernier ne fait pas partie 
des deux principaux critères du chercheur, il semble cependant revêtir beaucoup d'importance aux yeux des élèves. La théorie d'Henriot (1969) selon laquelle un dispositif au sein duquel l'utilisateur ne trouve pas d'accroche ludique n'est pas perçu comme un jeu par ce dernier prend ainsi toute sa valeur. Pour certains, le jeu permet même d'expérimenter le flow (Csíkszentmihályi, 2004), cet état d'engagement intense dans l'activité, souvent évoqué dans les théories du jeu.

Comme tous les jeux, *j'peux jouer des heures, *j'sais pas euh, on voit pas le temps passer. (Ivanhoé, T2: 47)

Le jeu renvoie à des modèles connus dans l'esprit des élèves, tels que les jeux d'aventure ou de guerre, et ils semblent s'attendre à en trouver les caractéristiques comme des missions ou des quêtes à accomplir.

Les deux caractéristiques principales évoquées par Brougère (2005), à savoir le second degré et l'espace décisionnel, sont également présents dans les représentations des apprenants.

[...] C'est mieux que ce soit pas un truc euh tout carré, euh tout euh, bien historique comme il faut, carré, etc. C'est bien d'apporter quelque chose d'un peu, un peu jeu vidéo euh, voilà. (Adrien : 47 - à propos du côté « science-fiction » de l'introduction dans l'environnement)

Enfin après ça reste un jeu. C'est à nous de le trouver [...]. (Katia, $\mathrm{T} 2: 118$ - à propos de la mission)

Katia trouve normal que, dans un jeu, le joueur ait à chercher et à trouver des solutions par lui-même. Il serait intéressant de réussir à déterminer si elle envisage le même type de démarche pour l'apprentissage.

Pour finir, quelques élèves se concentrent sur des contraintes qui leur semblent inhérentes au jeu vidéo. Celui-ci doit être facilement compréhensible et, dans ce cas, le choix de la langue peut devenir un obstacle. 
Ben je me concentrais plus sur la voix [I : Mmm.] pour essayer de comprendre quelque chose que (rire) qu'autre chose. (Quentin, T1 : 49 - à propos du fait qu'il ne s'est pas amusé)

Pour Quentin, par exemple, les difficultés de compréhension ont pris le pas sur tout le reste.

Pour d'autres, l'environnement dans lequel ils se trouvent est nécessaire pour considérer qu'ils se trouvent face à un jeu.

Parce que d'habitude les jeux vidéo, enfin j'y joue pas souvent, mais d'habitude c'est pas le même cadre. [...] Ben par exemple là on était debout. C'était en $3 \mathrm{D}$ déjà, y avait tout un matériel à mettre sur soi, et d'habitude ce n'est pas forcément le cas. (Monique, T2 : 10-11)

Nous retrouvons ainsi dans le discours des élèves un certain nombre de critères qui confirment ce que plusieurs théoriciens disent du jeu : second degré, liberté de choix, frivolité, attitude ludique, voire flow. Mais nous observons également que certains critères en relation avec les contenus ou le contexte d'apprentissage constituent des contre-arguments pour caractériser le jeu.

\subsection{Dénomination du dispositif}

Avant de nous concentrer sur la réponse à la question (a), nous avons effectué une recherche dans le but de savoir si le vocable « jeu » était utilisé pour désigner le dispositif sans que les apprenants y aient été incités.

Nous constatons (voir Figure 1) que, dans le cas du M1, les élèves ne font pas référence, sauf exception, à un jeu sans y être incités. Par ailleurs, les deux élèves qui parlent d'emblée de « jeu » ne désignent pas directement le dispositif en ces termes, mais l'associent à l'immersion dans l'environnement virtuel.

On a l'impression d'être dedans en fait, de pouvoir vraiment toucher les choses presque. Et euh, c'est mieux que dans les jeux vidéo par exemple où on sait très bien qu'on n'est pas dedans. (Christian, T1:2) 


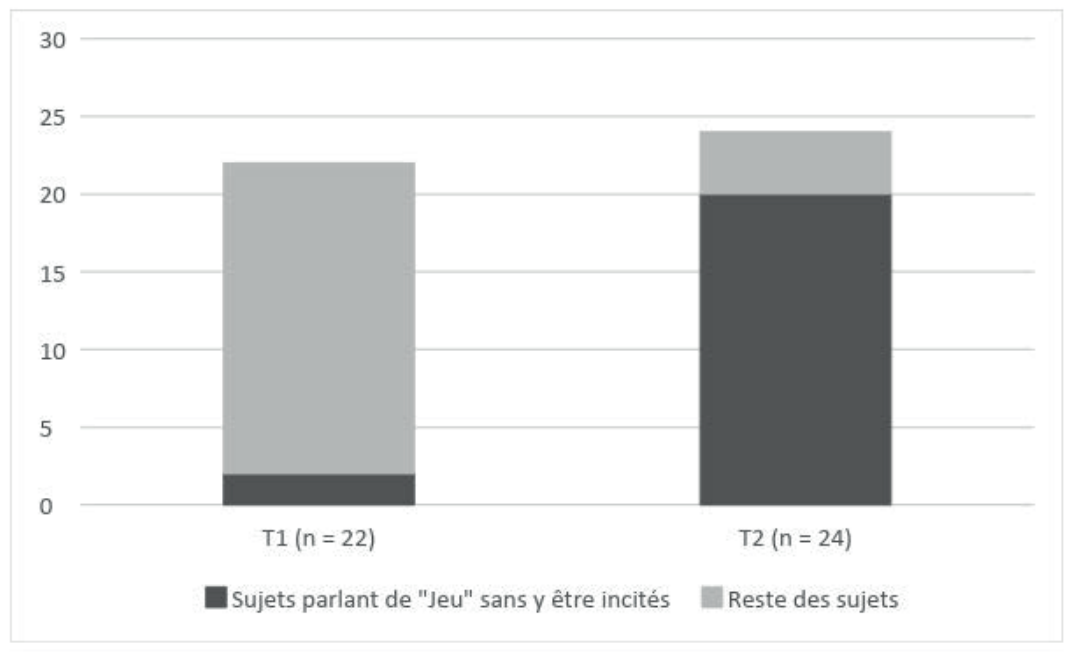

Figure 1. Désignation du dispositif avant la question (a)

Cette observation initiale laisse accroire que M1 n'a pas été perçu comme un jeu. Au contraire, durant T2, 20 élèves sur 24 qualifient M2 de « jeu » dès le début de l'entretien. Il semble ainsi que le dispositif M2 ait réellement été perçu comme un jeu, ce qu'une analyse des réponses à la question (a) tend à confirmer'.

Les élèves ayant testé M1 emploient des termes variés pour désigner le dispositif, ces derniers renvoyant à quatre champs lexicaux : ceux, dans l'ordre de prépondérance, du jeu, de l'exercice ou du cours, de l'expérimentation et de l'expérience immersive. Les qualificatifs utilisés par les élèves de T2 pour parler de M2 se regroupent dans trois catégories : le jeu, la simulation et l'activité sérieuse. Nous ne nous intéressons ici qu'aux réponses explicitant le choix du mot « jeu » ou expliquant le refus de désigner le dispositif comme tel.

4 La section qui suit jusqu'au Tableau 3 représente une synthèse de l'étude effectuée au cours du doctorat (voir Schmoll, 2016a, pp. 234-245 pour M1 et pp. 313-320 pour M2). 


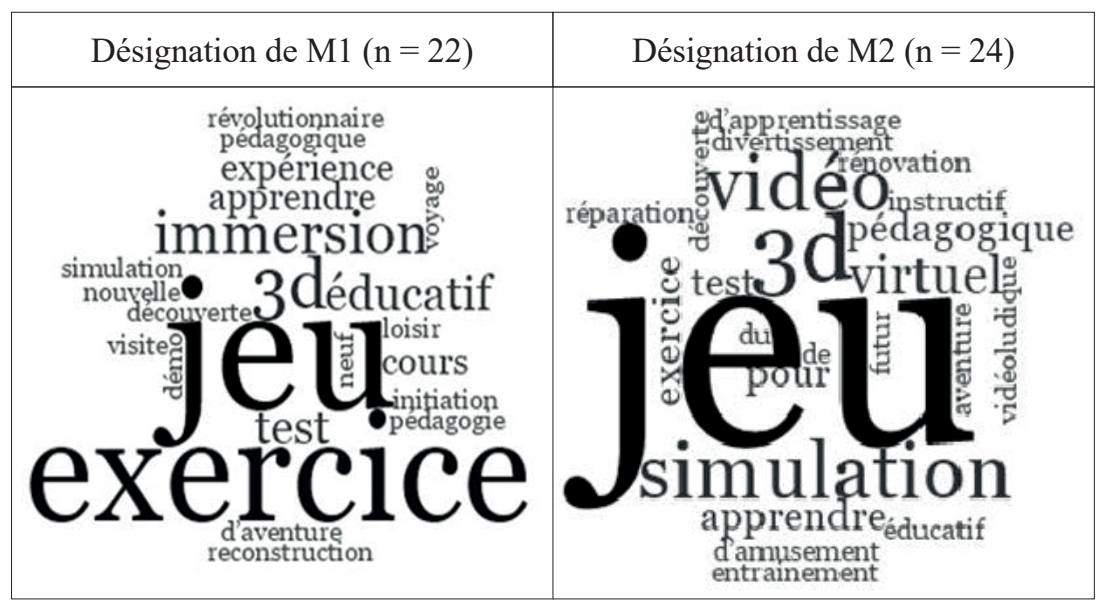

Figures 2 et 3. Désignation des deux dispositifs M1 et M2 par les élèves en réponse à la question (a)

Il est à noter tout d'abord que les élèves emploient rarement le substantif de façon isolée, précisant ainsi le domaine associé au jeu.

Tableau 2. Domaines associés au jeu dans le discours des élèves

\begin{tabular}{|c|l|c|c|}
\hline Domaines & Expansions du nom & T1 & T2 \\
\hline \multirow{4}{*}{ Domaine éducatif } & Jeu éducatif & 3 & 1 \\
\cline { 2 - 4 } & Jeu pour apprendre & 2 & 2 \\
\cline { 2 - 4 } & Jeu pédagogique & 1 & 2 \\
\cline { 2 - 4 } & Jeu d'apprentissage & - & 1 \\
\cline { 2 - 4 } & Jeu instructif & - & 1 \\
\hline \multirow{2}{*}{ Domaine technique } & Jeu 3D & - & 5 \\
\cline { 2 - 4 } & Jeu virtuel & - & 3 \\
\hline \multirow{2}{*}{ Domaine ludique } & Jeu vidéo (ludique) & - & 6 \\
\cline { 2 - 4 } & Jeu d'aventure & 1 & - \\
\hline Domaine de l'expérimentation & Jeu test & 1 & - \\
\hline Domaine de la nouveauté & Jeu neuf & 1 & - \\
\hline
\end{tabular}


Le jeu semble être majoritairement associé à l'apprentissage ; l'intention pédagogique des concepteurs apparaît ainsi explicitement dans l'esprit des élèves. Il est ensuite évoqué dans sa dimension technique, ce qui confirme l'importance que revêt le dispositif technique aux yeux des apprenants. La dimension ludique du jeu n'apparaît que dans un troisième temps.

Les explications données par les sujets quant au choix du qualificatif permettent d'apporter quelques précisions synthétisées dans le tableau 3. Nous reprenons les catégories du tableau 1, afin d'observer si nous retrouvons les mêmes attributs dans le discours des élèves.

Tableau 3. Représentations associées au mot jeu dans les réponses à la question (a)

\begin{tabular}{|c|c|c|c|c|}
\hline Catégories & $\begin{array}{c}\text { Nombre } \\
\text { d'occurrences } \\
\text { (tab. 1) } \\
\end{array}$ & Attributs & $\begin{array}{l}\text { Élèves } \\
\text { de T1 }\end{array}$ & $\begin{array}{l}\text { Élèves } \\
\text { de T2 }\end{array}$ \\
\hline $\begin{array}{l}\text { Technique / } \\
\text { tecnologique } \\
\text { ( } 9 \text { occurrences) }\end{array}$ & 16 & $\begin{array}{l}\text { Interactif, 3D, immersif, } \\
\text { effets spéciaux, écran }\end{array}$ & 3 & 6 \\
\hline $\begin{array}{l}\text { Divertissement } \\
\text { (11 occurrences) }\end{array}$ & 10 & Amusant, ludique, plaisir & 2 & 9 \\
\hline \multirow{3}{*}{$\begin{array}{l}\text { Genre et game } \\
\text { ou level design } \\
\text { (12 occurrences) }\end{array}$} & \multirow[t]{3}{*}{5} & $\begin{array}{l}\text { Enquêtes, missions, quêtes, } \\
\text { action, énigmes }\end{array}$ & 1 & 7 \\
\hline & & Violence, guerre & - & 1 \\
\hline & & $\begin{array}{l}\text { Objectifs à atteindre, } \\
\text { niveaux, incarnation d'un } \\
\text { personnage }\end{array}$ & - & 3 \\
\hline \multirow[t]{2}{*}{$\begin{array}{l}\text { Accessibilité } \\
(6 \text { occurrences })\end{array}$} & & $\begin{array}{l}\text { Compréhensible (langue), } \\
\text { allemand au sens négatif }\end{array}$ & 2 & 1 \\
\hline & & $\begin{array}{l}\text { Infantilisant, ennui } \\
\text { (scénario) }\end{array}$ & 2 & 1 \\
\hline $\begin{array}{l}\text { Public } \\
\text { (0 occurrence) }\end{array}$ & 4 & - & - & - \\
\hline $\begin{array}{l}\text { Fictionnel } \\
\text { (4 occurrences) }\end{array}$ & 2 & Virtuel, pas la réalité & - & 4 \\
\hline
\end{tabular}




\begin{tabular}{|l|c|l|c|c|}
\hline $\begin{array}{l}\text { Liberté } \\
\text { (5 occurrences) }\end{array}$ & 2 & $\begin{array}{l}\text { Liberté de choix (dans le } \\
\text { jeu ou de faire le jeu) vs } \\
\text { consignes }\end{array}$ & 3 & 1 \\
\cline { 2 - 5 } & $\begin{array}{l}\text { Liberté de déplacement, } \\
\text { mouvement }\end{array}$ & 1 & - \\
\hline $\begin{array}{l}\text { Environnement } \\
\text { (1 occurrence) }\end{array}$ & 2 & $\begin{array}{l}\text { Présences d'adultes, pas dans } \\
\text { son salon }\end{array}$ & 1 & - \\
\hline $\begin{array}{l}\text { Réflexion, app- } \\
\text { prentissage } \\
\text { (2 occurrences) }\end{array}$ & 0 & $\begin{array}{l}\text { Apprendre en s'amusant, } \\
\text { réfléchir à la langue, déduire } \\
\text { par soi-même }\end{array}$ & - & 5 \\
\hline $\begin{array}{l}\text { Absence de } \\
\text { caractéristiques } \\
\text { scolaires } \\
\text { (3 occurrences) }\end{array}$ & 0 & $\begin{array}{l}\text { Absence d'évaluation, } \\
\text { absence de vocabulaire } \\
\text { inconnu, tout à l'oral } \\
\text { (absence d'écrit) }\end{array}$ & - & 3 \\
\hline
\end{tabular}

Toutes les catégories relevées dans le tableau 1 sont présentes, en dehors de celles relatives au public. Les élèves associent toujours fortement le dispositif technique à la notion de jeu. La sensation de se divertir prend cependant plus de place dans le discours des sujets, surtout concernant ceux de T2. Ceci semble s'expliquer par le fait que M2 se rapproche d'un genre de jeu qu'ils connaissent comme les jeux de puzzle, d'aventure, d'énigme ou d'action et qu'ils y voient un véritable enjeu.

Ben c'est ce que c'est, c'est amusant quoi. Enfin faut répondre à, faut faire comme une sorte de quête. C'est comme résoudre des énigmes et tout. C'est, c'est bien. (Riwan, T2 : 29)

Le fait également que les apprenants trouvent des similarités en termes de game design avec les jeux vidéo les conduit à les considérer comme tels.

Nous retrouvons aussi les deux critères caractérisant le jeu de Brougère (2005). Le caractère fictionnel (second degré) est mis en relation avec le jeu par les élèves de T2 qui apprécient d'entrer dans un autre environnement. De leur côté, les sujets de T1 reprochent à M1 l'absence de liberté (espace décisionnel), ce qui n'est pas présent dans le discours des apprenants de T2. 
Parce qu'on est guidé, parce que par exemple dans un jeu vidéo, on fait euh, certes on a des missions ou ce genre de choses, mais là on avait vraiment quelque chose qui nous était dicté et qu'on devait faire. (Daniel, T1:33)

Il semble donc que la structure du scénario, laissant plus ou moins de liberté aux utilisateurs, joue un rôle déterminant dans les représentations des élèves concernant le jeu. Par ailleurs, une différence par rapport au discours général sur le jeu réside dans la vision du dispositif proposé, jugé par certains comme étant infantilisant en raison d'un gameplay trop simple.

La question de la compréhension langagière est évoquée par plusieurs élèves qui jugent que de devoir réfléchir en allemand annule le caractère potentiellement ludique du dispositif, d'autant plus que cela peut les empêcher de comprendre le scénario. Cependant, les élèves de T2 portent un regard différent sur le dispositif, certains faisant référence à un apprentissage vécu de façon positive grâce au jeu et jugeant l'absence de certaines caractéristiques scolaires comme un atout en faveur de l'expérience ludique et d'une sensation de plaisir.

$[\ldots]$ on peut s'amuser mais en même temps $[\ldots]$ apprendre des nouvelles choses en allemand [...]. (David, T2 : 24)

Le contexte scolaire ne semble, quant à lui, avoir que peu d'effet sur les représentations des élèves, seul un d'entre eux y faisant référence.

Pour conclure, si le dispositif est considéré avant tout comme un jeu de type éducatif, cela ne semble pas influencer le jugement favorable des élèves de T2, car M2 semble respecter certains critères du jeu comme le second degré et l'espace décisionnel, au contraire de M1. Un certain nombre de remarques de la part des apprenants quant à la présence de l'allemand nous invitent cependant à nous intéresser à la question (b). 


\subsection{Un jeu pour l'apprentissage de l'allemand}

La figure 4 représente les réponses des élèves à la question (b) : Que penses-tu de cette idée pour l'apprentissage de l'allemand?

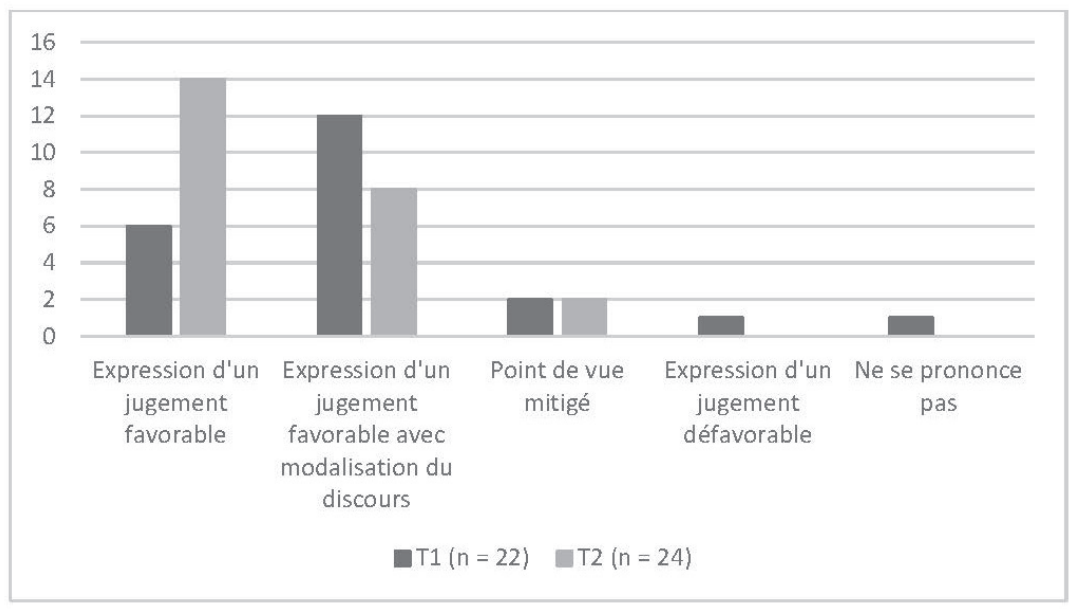

Figure 4. Réponses à la question (b)

Vingt sujets, essentiellement les élèves de T2, répondent sans équivoque à l'aide d'adjectifs ou d'adverbes mélioratifs : «(super) bonne idée », « intelligent », « bien », « mieux ». Un tel dispositif selon eux donne envie d'apprendre et est plus attirant que les cours. Il permet l'apprentissage de l'allemand en s'amusant, ainsi qu'un entraînement à la compréhension et à la production orales.

Les sujets de T1 expriment également majoritairement un avis favorable, mais davantage modalisé par des adverbes (" plutôt », « quand même ", « pas mal»), l'emploi du conditionnel ou encore des verbes modaux exprimant la possibilité (« ça peut », « ça doit être »). Les élèves montrent ainsi qu'ils adhèrent à l'idée, mais à certaines conditions : plus de complexité dans les actions, plus d'interactivité, plus de vocabulaire, etc. Les difficultés de compréhension langagière représentent notamment un frein majeur. 
Quatre élèves donnent un avis véritablement mitigé en trouvant à la fois des qualités et des défauts au dispositif et un sujet ne se prononce pas.

Seul Patrice exprime un avis défavorable $(\mathrm{T} 1: 42):$ « je pense que, à force, ça deviendrait un peu nul ». Cette déclaration met en valeur le fait que le dispositif ludo-éduquant représente une alternative potentiellement pertinente aux cours plus classiques, à condition de doser avec prudence son utilisation.

Nous constatons ainsi une relative unanimité quant à l'emploi d'un dispositif se voulant vidéoludique pour l'apprentissage de l'allemand. Le problème majeur identifié par la présente recherche concerne la compréhension de la langue-cible qu'il s'agit donc de particulièrement bien penser au cours de la conception.

\section{Conclusion}

Parce que nous nous positionnons dans un paradigme de centration sur l'individu (sur le joueur, sur l'apprenant), nous avons souhaité, dans cette étude, nous focaliser sur le discours des élèves au sujet de l'expérience qu'ils viennent de vivre, de ce qu'ils pensent du dispositif proposé et du contexte dans lequel ils se trouvent. Cette analyse de contenu portant sur des entretiens post-immersion nous a permis, à travers le relevé et l'analyse des termes qu'ils emploient pour désigner le dispositif expérimenté, de faire émerger les représentations des élèves sur le jeu vidéo en général et sur le jeu d'apprentissage en particulier. Ce choix méthodologique nous a semblé être un moyen privilégié pour tenter de mieux comprendre la bonne ou la mauvaise réception des jeux vidéo d'apprentissage, ainsi que les facteurs potentiellement favorables à l'émergence d'une attitude ludique et/ou d'une implication plus forte dans l'activité. L'analyse qualitative permet de faire émerger des pistes de réflexion et des questionnements utiles, notamment dans une perspective d'amélioration du produit expérimenté.

Les résultats de cette étude mettent en exergue que, plus que le contexte scolaire, très peu présent dans le discours des élèves, c'est le dispositif proposé qui influence leurs représentations. 
Tout d'abord, au regard des nombreuses considérations techniques exprimées au cours des entretiens, le dispositif technique semble revêtir une coloration ludique à leurs yeux. L'immersion tridimensionnelle semble faciliter l'entrée dans un espace-temps fictionnel que l'on peut rapprocher de la notion de second degré du jeu établie par Brougère (2005). Cette expérience immersive et l'intérêt qui lui est associé semblent même exercer une influence positive sur les représentations liées à l'apprentissage de certains apprenants. L'état émotionnel et/ou la motivation générés par le dispositif joueraient un rôle cognitif d'après ces derniers. Si le dispositif 3D, associé à l'expérience ludique, permet à certains élèves de se sentir complètement engagés dans l'activité (flow), il pourrait alors y avoir des effets positifs en termes d'apprentissage tels que ceux décrits par Csíkszentmihályi (2004, p. 77) : une « meilleure performance », le « développement des capacités », ainsi que de l' « estime de soi ». Ceux-ci nécessitent cependant une analyse plus poussée. Par ailleurs, l'effet de nouveauté que produit le caractère inhabituel de l'équipement explique qu'il soit si présent dans leur discours. Guichon (2012, section 3.2.2.2, para. 41) souligne à ce sujet le risque que cet attrait soit temporaire, les outils générant un " effet de halo autour de l'apprentissage ». Nous pouvons ainsi nous demander si, une fois l'effervescence passée, les élèves manifesteront toujours autant d'intérêt face au dispositif, comme le déclare Patrice dans la section précédente. Le dispositif technique seul n'est pas suffisant, à notre sens, pour provoquer chez les apprenants un intérêt, voire une motivation, à long terme.

Par ailleurs, l'aspect fermé et prescriptif de M1 est ce qui semble avoir le plus marqué les apprenants de T1, les empêchant de considérer le dispositif comme un jeu. C'est l'absence de liberté et l'impossibilité de prendre des décisions qui semblent empêcher l'émergence d'une attitude ludique. Au contraire, avec le scénario M2, les apprenants, qui devaient prendre des initiatives, semblent avoir éprouvé davantage de plaisir à employer le dispositif, à tel point que nous trouvons dans leurs propos des énoncés positifs associant le jeu et l'acte d'apprendre. Ce constat concorde avec le deuxième critère évoqué par Brougère (2005) pour caractériser le jeu. Ce dernier, pour être considéré comme tel, doit pouvoir 
laisser la possibilité au sujet de prendre des décisions qui auront un effet sur la réussite ou l'échec de la partie. Sans cet élément, l'utilisateur ne peut pas investir l'objet ou l'activité et peut donc difficilement s'impliquer. Ces propos rejoignent le discours tenu par certains théoriciens de la motivation. En effet, un des deux facteurs censés influencer la motivation intrinsèque correspond au « locus de causalité perçu » (Vallerand \& Thill, 1999, p. 262). Le fait ou l'impression d'avoir le choix, de pouvoir prendre des décisions, provoque un sentiment d'autodétermination nécessaire à la motivation. Cette perspective conforte l'idée qu'il est loin d'être suffisant de proposer un jeu à des élèves pour provoquer automatiquement chez eux une attitude ludique et un engagement sans faille. Pour finir, l'intégration d'un dispositif vidéoludique à visée pédagogique en milieu scolaire semble, d'après notre étude, obtenir un avis majoritairement favorable de la part des élèves, ce qui confirme, tout en y apportant des précisions utiles, d'autres études menées auparavant qui s'intéressaient au point de vue général des apprenants (Wix, 2012) ou à celui des enseignants (Wastiau, 2009).

Nous pouvons néanmoins nous interroger sur sa pertinence du point de vue cette fois du concepteur ou de l'enseignant. L'implication possible que peuvent manifester les élèves en début de parcours, si le dispositif ludo-éduquant est jugé positivement, a-telle des chances de perdurer ou va-t-elle s'estomper dès lors que l'effet de nouveauté disparaît ? Et, au-delà de cette implication, quels sont véritablement les gains en termes d'apprentissage ou de rétention des connaissances ? L'enquête menée par Wastiau (2009), tout comme le rapport de Wix (2012), en présentent certains mais ceux-ci reposent sur les impressions des enseignants et non pas sur des données mesurables. Il reste donc une étude longitudinale à mener sur ce sujet qui n'est pas sans poser un certain nombre de problèmes méthodologiques. 


\section{Références}

Alvarez, J. (2007). Du jeu vidéo au Serious game. Approches culturelle, pragmatique et formelle. Thèse de Doctorat, Université de Toulouse II et III. Disponible à : http:// ja.games.free.fr/These_SeriousGames/TheseSeriousGames.pdf.

Anderson, J. A. (1988). Examen de quelques concepts éclairant la position de l'éducateur aux médias. Actes du Symposium de Lausanne 23, 11-23.

Brougère, G. (2002). Jeu et loisir comme espaces d'apprentissages informels. Éducation et sociétés 10, 5-20.

Brougère, G. (2005). Jouer / Apprendre. Paris : Economica.

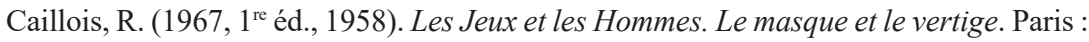
Gallimard.

Conseil de l'Europe (2001). Un cadre européen commun de référence pour les langues : apprendre, enseigner, évaluer. Strasbourg: Division des Politiques linguistiques.

Csíkszentmihályi, M. (2004, $1^{\text {re }}$ éd. : 1990). Vivre. La psychologie du bonheur. Paris : Robert Laffont.

Genvo, S. (2012). La théorie de la ludicisation : une approche antiessentialiste des phénomènes ludiques. Jeu et jouabilité à l'ère numérique. Disponible à : http://www. ludologique.com/publis/LudicisationDec12.pdf.

Guichon, N. (2012). L'apprentissage des langues médiatisé par les technologies (ALMT) - Étude d'un domaine de recherche émergent à travers les publications de la revue Alsic. Alsic 15(3). Disponible à : http://alsic.revues.org/2539.

Henriot, J. (1969). Le jeu. Paris : Synonyme.

Koster, R. (2005). A Grammar of Gameplay - Game Atoms: Can Games Be Diagrammed? Disponible à : http://www.raphkoster.com/gaming/atof/grammarofgameplay. pdf.

Meunier, J.-P. \& Peraya, D. (2010). Introduction aux théories de la communication. Bruxelles : De Boeck.

Pädagogische Hochschule Karlsruhe (2011). Formulaire de demande de concours communautaire. ÉVEIL 3D, version déposée (Réf. : 4106) (juin 2013). Strasbourg : Non publié.

Peterson, M. (2013). Computer Games and Language Learning. New York : Palgrave Macmillan.

Puren, C. (2009). Variations sur la perspective de l'agir social en didactique des langues-cultures étrangères. Disponible à : http://www.aplv-languesmodernes.org/ spip.php?article1888.

Schmoll, L. (2016a). Concevoir un scénario de jeu vidéo sérieux pour l'enseignement-apprentissage des langues ou comment dominer un oxymore. Thèse de doctorat, Université de Strasbourg. Disponible à : https://tel.archives-ouvertes.fr/tel01511336/document.

Schmoll, L. (2016b). L'emploi des jeux dans l'enseignement des langues étrangères : Du traditionnel au numérique. Sciences du jeu 5. Disponible à : http://sdj.revues. org $/ 628$.

Schmoll, L. (2017). Penser l'intégration du jeu vidéo en classe de langue. Jeux et langues dans l'enseignement supérieur, Recherche et pratiques pédagogiques en langues de spécialité 36(2). Disponible à : http://apliut.revues.org/5722. 
Sykes, J. M. \& Reinhardt, J. (2013). Language at Play. Digital Games in Second and Foreign Language Teaching and Learning. New Jersey, USA : Pearson.

Vallerand, R. J. \& Thill E. E. (1999). Introduction à la psychologie de la motivation. Laval, Québec : Éditions Études Vivantes.

Wastiau, P. (coord.). (2009). How are Digital Games Used in Schools? Bruxelles : European Schoolnet.

Wix, A. (2012). Jouer en classe, est-ce bien sérieux? Bilan de l'expérimentation académique sur les usages de jeux sérieux au collège et au lycée. Académie d'Aix Marseille. Disponible à : https://www.pedagogie.ac-aix-marseille.fr/upload/docs/ application/pdf/2012-10/bilan_jeux-serieux_2011-2012.pdf.

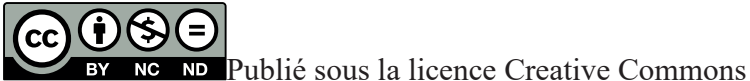

«Attribution - Pas d'Utilisation Commerciale - Pas de Modification 4.0 International» (CC BY-NC-ND) 